\title{
Energetic particle sounding of the magnetospheric cusp with ISEE-1
}

\author{
K. E. Whitaker, T. A. Fritz, J. Chen, and M. Klida \\ Center for Space Physics, Boston University, Boston, MA, USA \\ Received: 10 August 2006 - Revised: 14 April 2007 - Accepted: 7 May 2007 - Published: 4 June 2007
}

\begin{abstract}
Observations on 30 October 1978 show the ISEE1 spacecraft passing though the high-altitude dayside northern magnetospheric cusp region from roughly 16:00 to 18:30 UT, during a slow solar wind period $(\sim 380 \mathrm{~km} / \mathrm{s})$. More than two orders of magnitude enhancements of the cusp energetic particle (CEP) fluxes were observed along with a depressed and turbulent local magnetic field. The observed variations of the pitch angle distributions (PAD) provide a unique opportunity to determine the structure of the cusp and the origin of the CEP. Through a boundary sounding technique, the location and orientation of the cusp poleward (or backside) boundary was observed for almost 10 min during which time it appeared initially to be stationary in the GSM/GSE $\mathrm{X}$-direction and then moved sunward about 0.12 Earth radii $\left(R_{E}\right)$. The orientation remained approximately perpendicular to the GSM/GSE X-axis until it was observed to rotate by 60 degrees in $\sim 3$ min before ISEE- 1 was fully inside the cusp cavity. The cavity itself was filled with CEP fluxes displaying large anisotropies, indicative of their source being located below (Earthward) of the satellite location. The spacecraft entered from the backside of the cusp, then traveled $\sim 4 R_{E}$ through the cavity, and exited through the "top" of the cavity leaving a region of energetic ions below. The PADs demonstrate that the bow shock cannot be the main source of the observed CEPs. The CEP fluxes were measured at about $8.5 \mathrm{~h}$ MLT when the IMF had both an 8-10 nT duskward and southward component.
\end{abstract}

Keywords. Magnetospheric physics (Energetic particles, precipitating; Magnetopause, cusp, and boundary layers; Magnetospheric configuration and dynamics)

Correspondence to: K. E. Whitaker

(kwhitake@bu.edu)

\section{Introduction}

The magnetospheric cusp is defined by a combination of (1) a decrease in magnetic field magnitude in the dayside highlatitude region (Farrell and Van Allen, 1990) and (2) a more than one order of magnitude increase in intensity of the 1$10 \mathrm{keV}$ ions (Chen et al., 1997, 1998; Fung et al., 1997). Energetic particles are observed regularly in the cusp. However, the origin of the Cusp Energetic Particles (CEP) remains controversial. The CEPs have been suggested to have three possible sources, (1) the ions are accelerated to tens of $\mathrm{keV}$ at the bow shock and arrive in the cusp along a magnetically guided trajectory (Chang et al., 1998), (2) the CEPs are produced by substorms in the geomagnetic tail (Delcourt and Sauvaud, 1999; Blake, 1999; Antonova et al., 2000) and arrive in the cusp through a "Shabansky" orbit (Shabansky, 1971; Antonova and Shabansky, 1975), and (3) the electrons and ions are both accelerated locally up to energies of hundreds of keV to several MeV (Chen and Fritz, 1998; Chen et al., 1998).

Energetic particles in the magnetosphere can be used as remote sensors probing the distance and orientation of a trapping boundary (Konradi et al., 1965; Williams, 1979; Fritz and Fahnenstiel, 1982). The large gyroradii of energetic ions remotely probe boundaries up to distances greater than about $1000 \mathrm{~km}$ from the spacecraft.

The International Sun-Earth Explorer's ISEE-1 spacecraft was launched on 22 October 1977 into a highly eccentric $\left(1 \times 23 R_{E}\right)$ geocentric orbit, with an inclination of $28.76^{\circ}$ and a period of $\sim 57 \mathrm{~h}$. The Medium Energy Particle Experiment (MEPE) onboard ISEE-1 measured the Pitch Angle Distributions (PAD) of the energetic particles (Williams et al., 1978). Due to the low inclination of the orbit, the spacecraft has previously been thought to never have passed through the high-altitude dayside cusp region.

Observations on 30 October 1978 from roughly 16:0018:30 UT for an outbound pass of the ISEE-1 spacecraft

Published by Copernicus GmbH on behalf of the European Geosciences Union. 


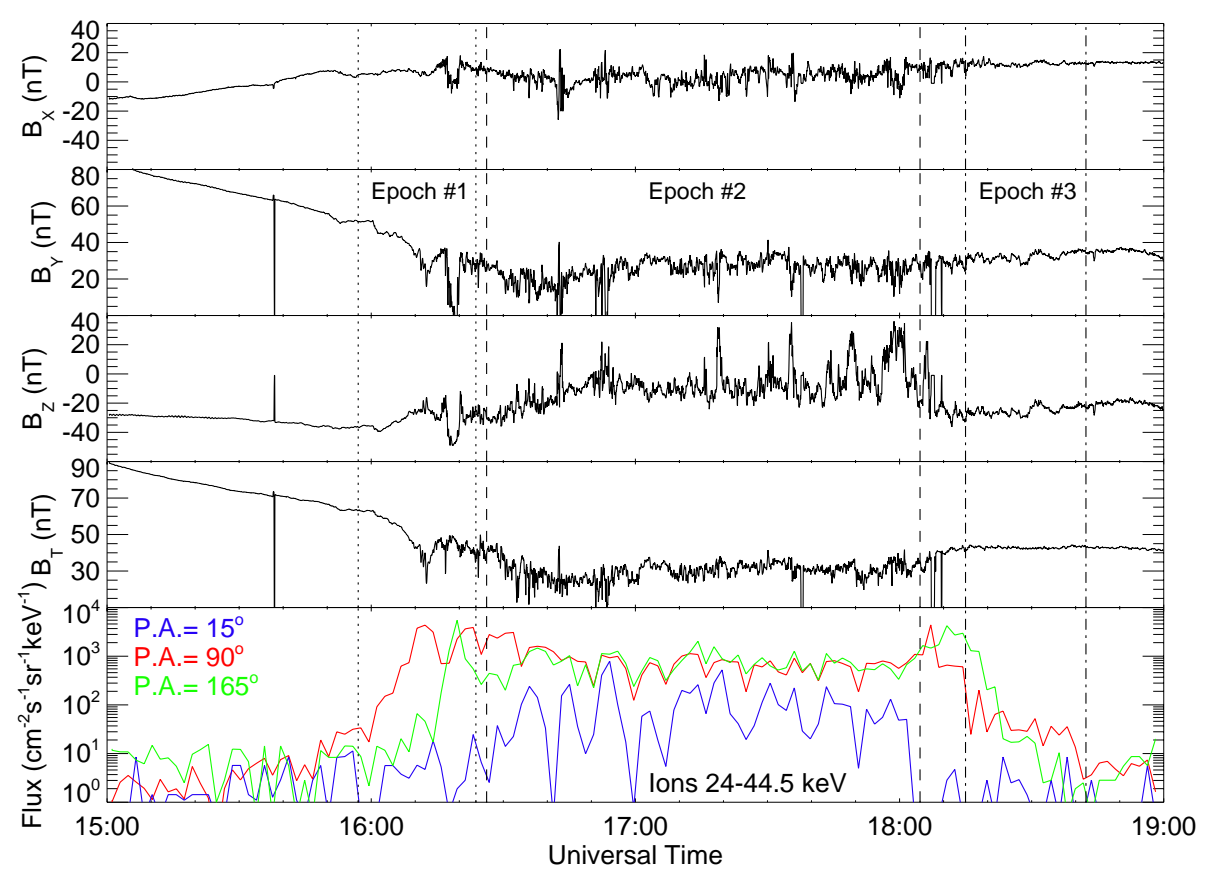

Fig. 1. The GSM $\hat{X}, \hat{Y}$, and $\hat{Z}$ magnetic field components (top 3 panels, respectively) and the total magnetic field (panel 4 ) observed by ISEE-1 from 15:00-19:00 UT on 30 October 1978. The bottom panel contains the proton flux for 24-44.5 keV energy channel, where the particles are sorted by their pitch angles, particle detected at a pitch angle of $15^{\circ}$ is blue, $90^{\circ}$ is red, and $165^{\circ}$ is green, with a tolerance of $\pm 5^{\circ}$. The dotted lines correspond to the time period for Fig. 3, dashed lines for Fig. 4, and dot-dashed line for Fig. 5 .

\section{Detector Look Direction}

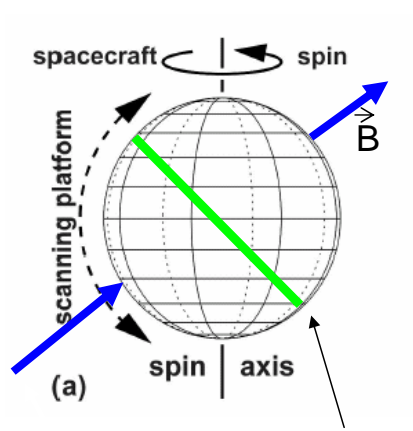

Locus of $\alpha=90^{\circ}$ pitch angles

Fig. 2. This figure demonstrates how one interprets Figs. 3-5. The detector scans through the unit sphere in $36 \mathrm{~s}$ (left) and the fluxes are linearly translated from elevation and azimuth onto an orthogonal Cartesian coordinate system (right). The green line corresponds to the locus of all $90^{\circ}$ pitch angles. The blue arrow is the magnetic field vector, where it points from the center of the $30^{\circ}$ contour to the center of the $150^{\circ}$ contour.

show cusp-like characteristics, including ion flux enhancements of over two orders of magnitude for over two hours and a depressed and turbulent local magnetic field and both ionospheric and solar wind plasma (Whitaker et al., 2006). The solar wind conditions during the observations were steady and slow $(\sim 380 \mathrm{~km} / \mathrm{s})$. During the CEP event period, the IMF $B_{y}$ was positive (duskward) and had a value of about 8-10 nT from 16:40-18:30 UT, while both IMF $B_{x}$ and $B_{z}$ were negative (tailward and southward). The local magnetic field observed by ISEE-1 (Fig. 1) had a negative $B_{z}$ component that fluctuated between $\sim 0$ and $-30 \mathrm{nT}, B_{x}$ was roughly $0 \mathrm{nT}$, and $B_{y}$ was positive $(\sim 30 \mathrm{nT})$. The most turbulent activity occurs in the Z-component. The three-dimensional particle pitch angle distributions can be used to explore the geometry and evolution of the cusp boundary as the spacecraft first penetrates the boundary, as well as when the spacecraft exits the cusp. The variation of $24-44.5 \mathrm{keV}$ ions during this interval is presented in the bottom panel of Fig. 1.

The energy range for CEP is from about $20 \mathrm{keV}$ to $10 \mathrm{MeV}$ (Chen et al., 1997, 1998; Fritz et al., 2003). Trattner et al. (2001) claimed that the CEPs with energies $<150 \mathrm{keV}$ were from the quasi-parallel bow-shock and that while those CEPs with energies $>150 \mathrm{keV}$ were from the radiation belt. The present study shows that the $24-44.5 \mathrm{keV}$ CEPs are also not from the bow shock. Based on low-altitude $\left(<2.2 R_{E}\right)$ cusp observations, Kremser et al. (1995) reported that the cusp ion populations were a mixture of ionospheric and solar wind particles. Asikainen and Mursula $(2005,2006)$ suggested that the CEPs were from the high-latitude (quasi) trapping region. 


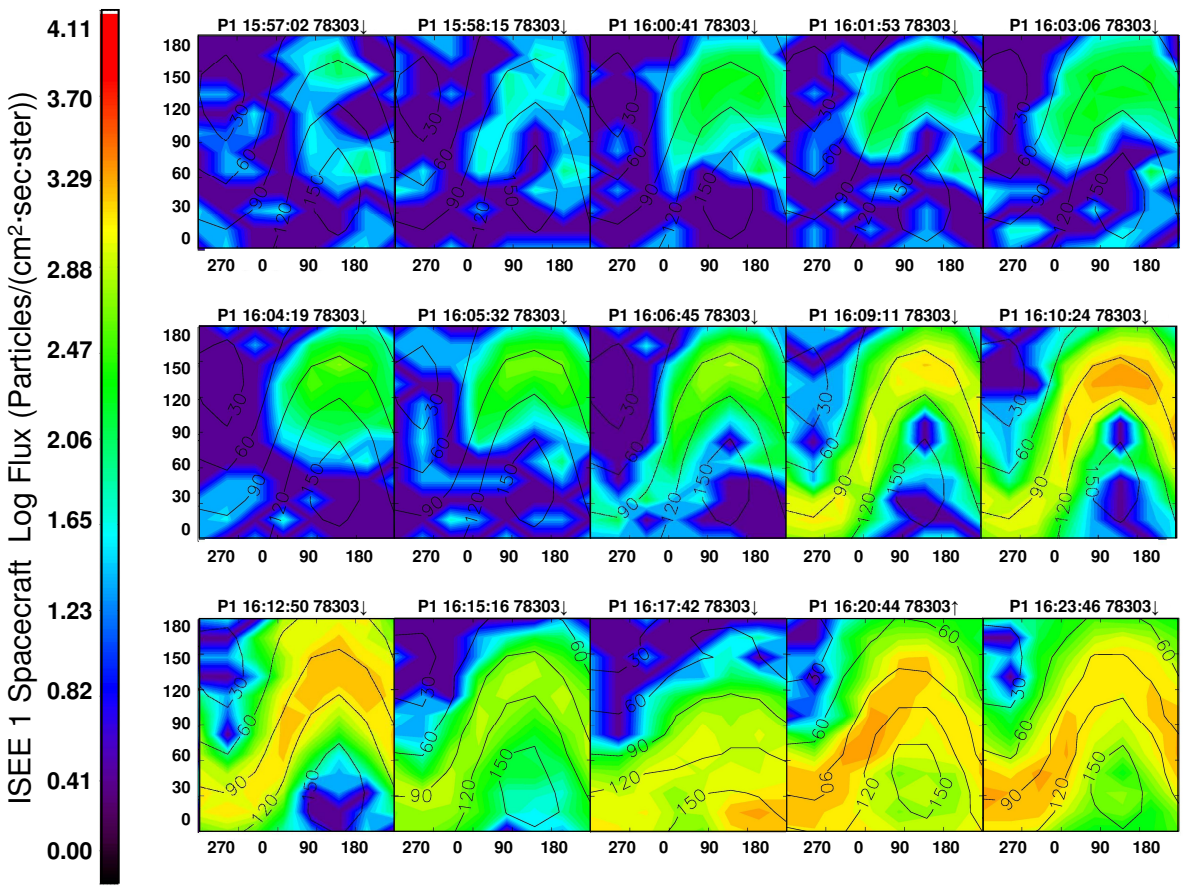

Fig. 3. ISEE-1 MEPE 3-D pitch angle distributions for the time period of 15:57-16:24 UT (Epoch 1 in Fig. 1). The s/c was fully engulfed in CEPs by 16:09 UT, where the particles with a PA of $90^{\circ}$ are seen at all azimuthal angles. The intense population from below the s/c appears around 16:15 UT.

The ISEE-1 observations of the cusp provide new insights into the CEP behavior and the geometry of the cusp through the three-dimensional PAD.

\section{Observations}

ISEE-1 passed through the high-altitude dayside cusp region on 30 October 1978 from roughly 16:00 to 18:30 UT at about $8.5 \mathrm{~h}$ local time. The ISEE-1 spacecraft traveled from 3.98.2 $R_{E} \hat{\mathrm{X}},-(5.0-8.2) R_{E} \hat{\mathrm{Y}}$, and 6.0-6.8 $R_{E} \hat{\mathrm{Z}}$ in GSM coordinates. In this paper, we investigate the geometry of the cusp boundary and the evolution of the boundary orientation angle through a boundary sounding technique as ISEE-1 penetrated into the cusp diamagnetic cavity (CDC) while observing intense CEP fluxes.

The ISEE-1 MEPE detector scans through the unit sphere in $36 \mathrm{~s}$ as illustrated in Fig. 2. The locus of particles with pitch angles of $90^{\circ}$ for the example PAD is traced on the unit sphere, with the magnetic field vector pointing from a PA of $0^{\circ}$ to $180^{\circ}$. Figure 2 is representative of how to interpret the PAD in Figs. 3-5, described below. The left panel of Fig. 2 is plotted for the fluxes in detector look direction, the direction from which the energetic particles are coming. The scale on the horizontal axis has the sunward direction at $0^{\circ}$ and the anti-sunward direction at $180^{\circ}$. The vertical axis is the scan direction, where $0^{\circ}$ is anti-parallel to the spacecraft spin axis, viewing the south ecliptic pole, and $180^{\circ}$ is parallel to the spin axis viewing the north ecliptic pole. At a (vertical) elevation of $90^{\circ}$, the $0^{\circ}$ and $90^{\circ}$ azimuth directions represent essentially the GSE X- and Y-axes, respectively.

\subsection{ISEE-1 MEPE energetic particle distributions}

Figures 3-5 contain three-dimensional energetic ion particle distributions with energies of $24-44.5 \mathrm{keV}$ (notice that the figures do not have the same color bars). The panels trace through the epoch where the spacecraft is penetrating the boundary of the cusp (Fig. 3), inside the CDC (Fig. 4), and just outside/above the cusp (Fig. 5). In Sect. 3, the panels in Fig. 3 are used to determine the distance to the boundary of the cusp and the orientation of this boundary.

As the spacecraft first penetrates the cusp around 15:57 UT, energetic particles peaked at $90^{\circ}$ are observed with azimuthal angles of roughly $45^{\circ}$ to $180^{\circ}$. From $16: 00$ to 16:05 UT, the azimuthal angle range slowly increases by $45^{\circ}$ to roughly $0^{\circ}$ to $225^{\circ}$. After $16: 05 \mathrm{UT}$, the particles begin to fill out and arrive from all azimuthal angles. By 16:08 UT the spacecraft observes strong particle populations peaked at 90 degrees (see Panel 9 in Fig. 3, 16:09:11 UT) at all azimuthal angles.

Around 16:15 UT, about $15 \mathrm{~min}$ after the spacecraft penetrated the boundary, a population of particles from below appears (see panel 13 in Fig. 3, 16:17:42 UT). This population 


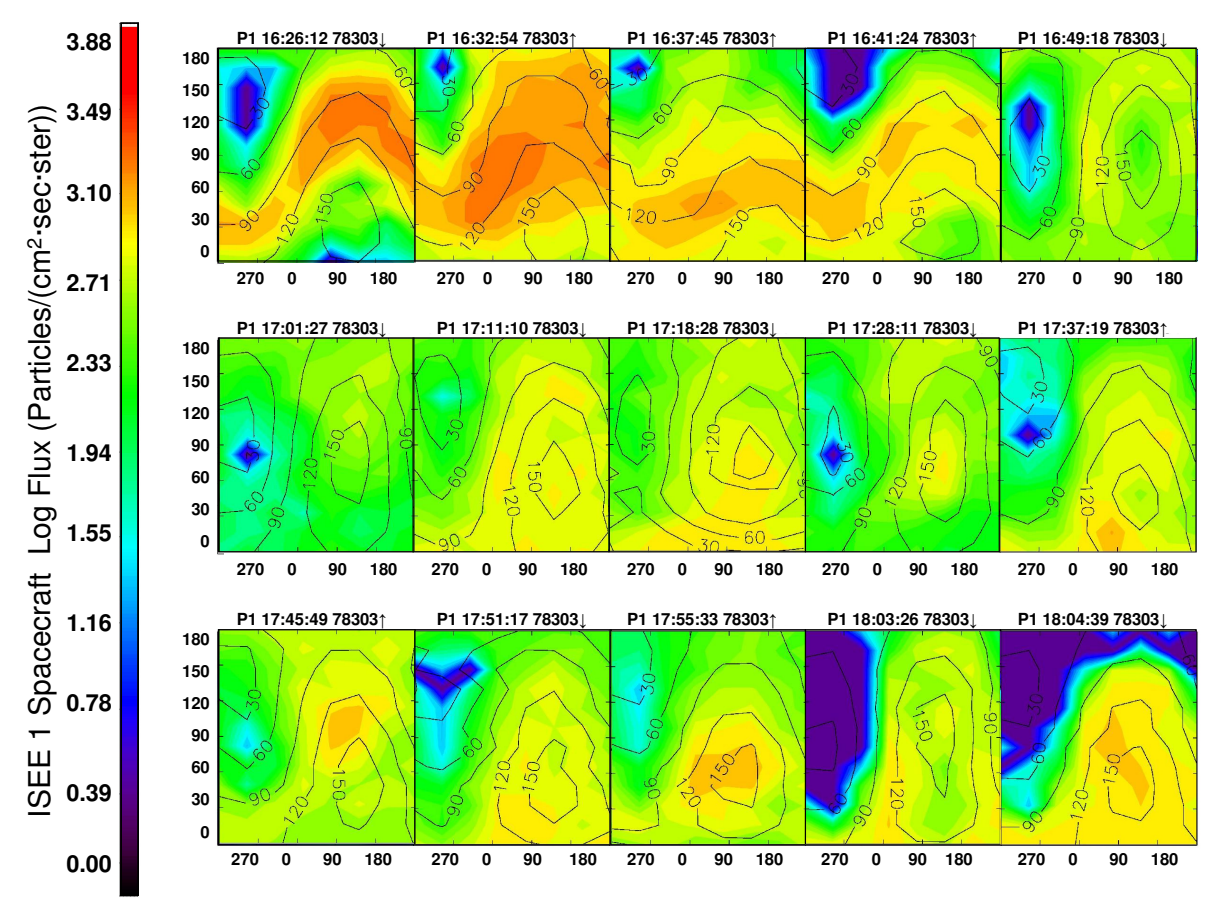

Fig. 4. ISEE-1 MEPE 3-D pitch angle distributions for the time period of 16:26-18:04 UT (Epoch 2 in Fig. 1). This epoch corresponds to when the $\mathrm{s} / \mathrm{c}$ is traveling through the CDC observing a very turbulent magnetic field, where the particles strongly follow the field lines.

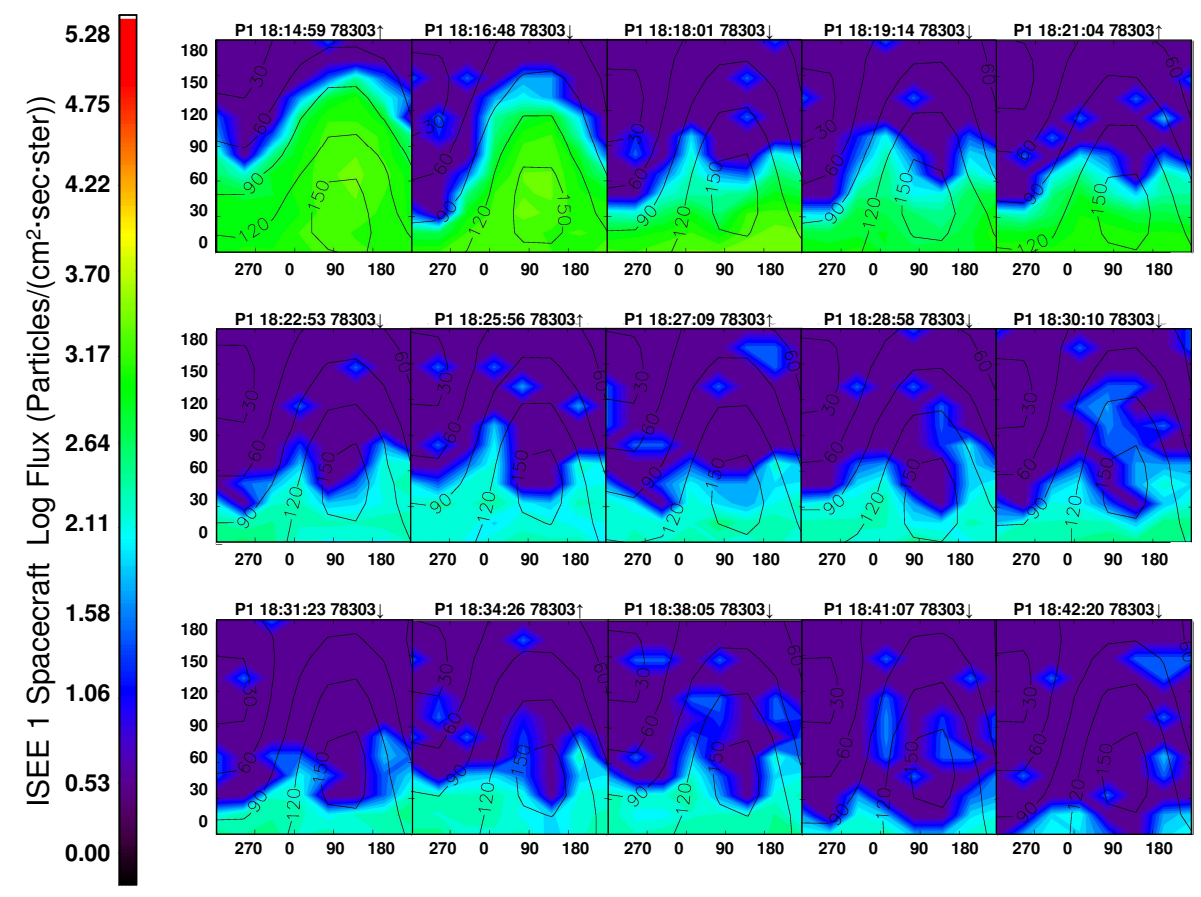

Fig. 5. ISEE-1 MEPE 3-D pitch angle distributions for the time period of 18:15-18:42 UT (Epoch 3 in Fig. 1). The s/c has now exited out the "top" of the CDC, but still observes energetic particles from below until 18:42 UT. 

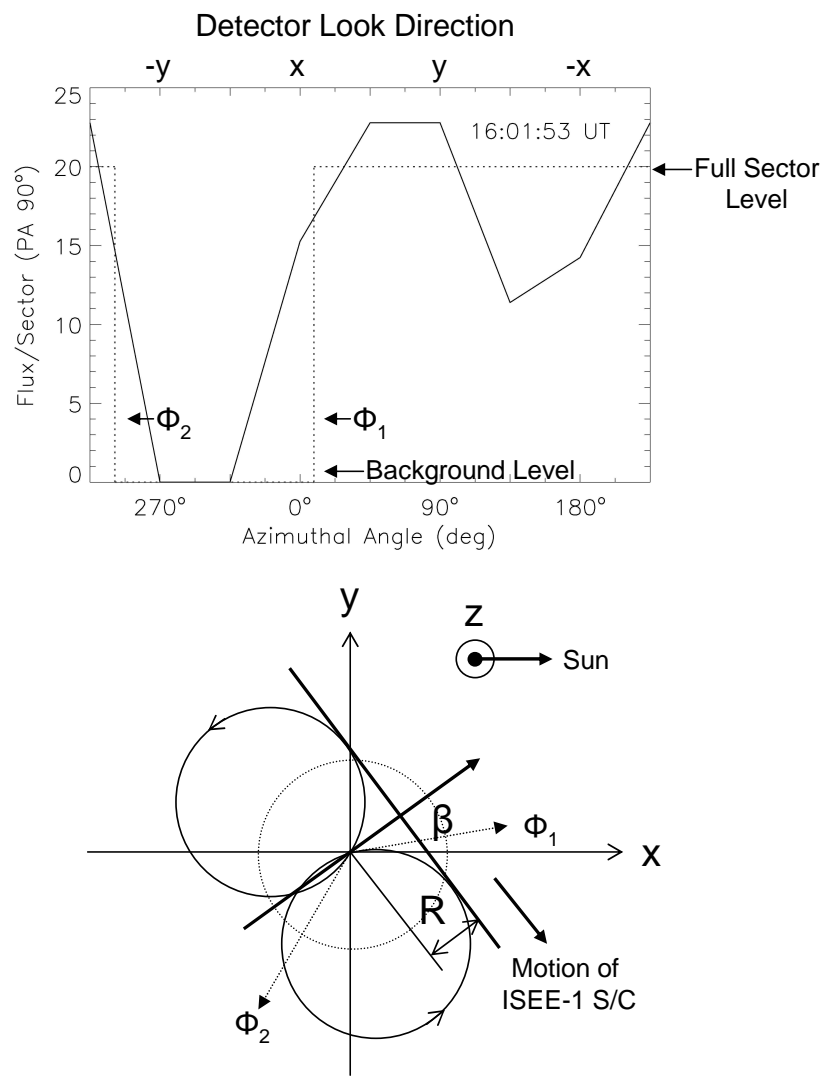

Fig. 6. The top plot shows the flux per sector for the 16:01:53 UT record, where there is an absense of particles at a range of azimuthal angles becauce the s/c is too far away from the CDC boundary to observe particles with those gyroradii. These angles are used to find the orientation angle $\beta$, measured from the $\mathrm{x}$-axis, and the distance $R$ of the boundary from the s/c (bottom). This method is used for Figs. 7-8.

dominates from 16:17-16:19 UT, after which time the particles remain enhanced but the $90^{\circ}$ particles dominate (Panels 14 and 15, Fig. 3).

While ISEE-1 is in the CDC, the spacecraft observes a strong population of particles filling the hemisphere below the sensor (Fig. 4). The magnetic field vector points from the center of the $30^{\circ} \mathrm{PA}$ contour $\left(\alpha=0^{\circ}\right)$ to the center of the $150^{\circ}$ PA contour $\left(\alpha=180^{\circ}\right)$. Although the magnetic field vector is variable, the particles closely follow the field lines.

After the ISEE-1 spacecraft has exited the cusp and no longer observes the characteristic turbulent and depressed magnetic field and ion flux enhancements, there remains a population of particles from below predominately with pitch angles of $120^{\circ}$ until 18:42 UT (see Fig. 5). The threedimensional PAD appear to be a reverse bite-out to the patterns observed in Fig. 3 as the spacecraft appears to penetrate the boundary of the cusp by moving above the CDC filled with CEP fluxes.

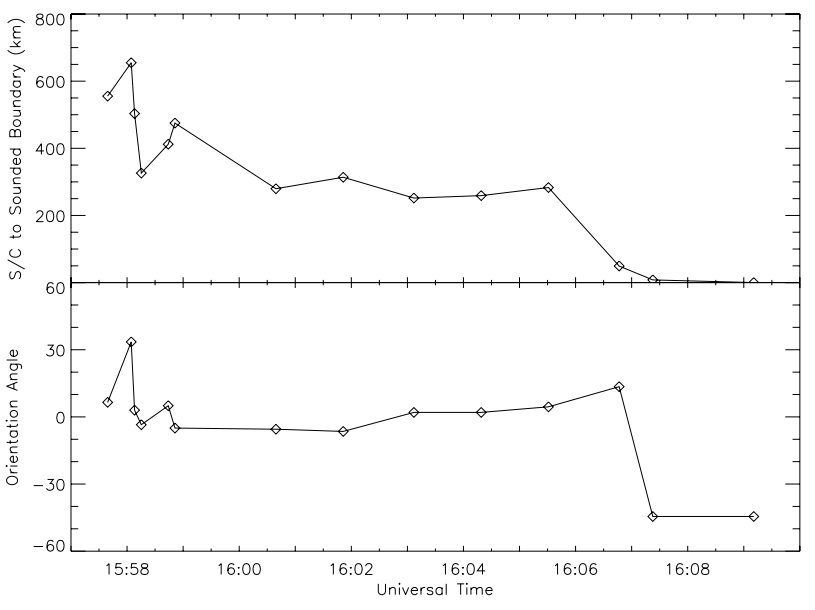

Fig. 7. Using Eqs. (1-2), the distance from the s/c to the sounding boundary $(\mathrm{km})$ and the orientation angle of the boundary is calculated for the entire time the s/c remotely senses the boundary.

\section{Boundary sounding technique}

As the ISEE-1 MEPE detector scans through the unit sphere with $B_{z}<0$, it will "see" particles arriving from directions counterclockwise between two angles, $\phi_{1}$ and $\phi_{2}$. As illustrated in Fig. 6, the remaining angles outside of this region ( $\phi_{2}$ to $\phi_{1}$ ) show no observed particles, because the gyrocenter is beyond one gyroradii from the emitting boundary. Previous work sounded absorbing boundaries from 2 gyroradii inside the magnetopause (Konradi et al., 1965; Williams, 1979; Fritz and Fahnenstiel, 1982), whereas this work sounds a particle source boundary from 2 gyroradii outside the emitting boundary. Figure 1 shows that around 16:00 UT on 30 October 1978, $B_{y}$ (GSE) was dominant, while Fig. 6 assumes the B vector was along the anti-parallel $\mathrm{Z}$ (GSE) direction at the time. Therefore, this remote sensing method assumes that an ion source boundary and the local B vector were along the anti-parallel Z (GSE) direction. Although a real boundary has a finite thickness and irregularities, the perpendicular distance $R$ and orientation angle $\beta$ to the plane of the boundary can be estimated from these angles, depending on the gyroradii $r_{g}$ of the measured particles. The gyroradius is determined for $24-44.5 \mathrm{keV}$ particles with a local magnetic field averaged over each $36 \mathrm{~s}$ scan of the unit sphere. The distance $R$ from the spacecraft to the emitting boundary is given by

$R=r_{g}\left(1+\cos \frac{\left(\phi_{2}-\phi_{1}\right)}{2}\right)$

The tilt angle of the boundary normal relative to the $\mathrm{X}$-axis is given by

$\beta=\frac{\left(\phi_{1}+\phi_{2}\right)}{2}-90^{\circ}$ 


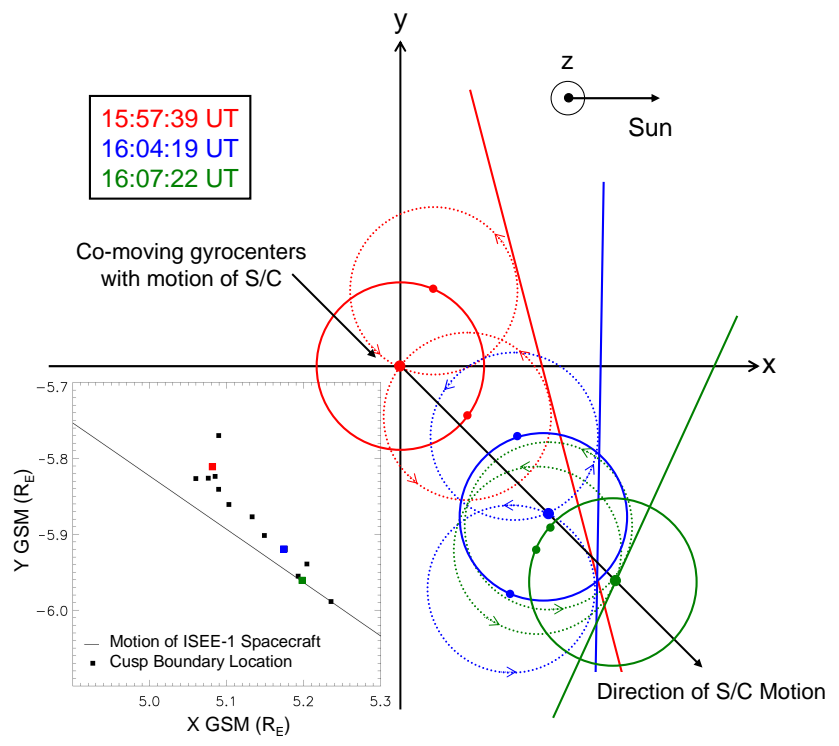

Fig. 8. An illustration of the evolution of the CEP boundary from 15:57-16:07 UT. The center of the gyroradii is assumed to be comoving with the motion of the spacecraft. The boundary is initially roughly parallel to the direction the $\mathrm{s} / \mathrm{c}$ is traveling, rotating about $60^{\circ}$ in $3 \mathrm{~min}$ so that the $\mathrm{s} / \mathrm{c}$ penetrates the CEP boundary perpendicular. The inset plot on the lower left shows the GSM X-Y position of the satellite (solid line) and a projected evolution of the boundary location from the data presented in Fig. 7 (squares).

In Fig. 3, as noted above, the flux enhancements are truncated along the $90^{\circ}$ pitch angle contour relative to the azimuthal angle. As the spacecraft penetrates the cusp traveling closer to the emitting boundary, the range of azimuthal angles where energetic particles are observed increases until the signature PAD peaked at $90^{\circ}$ through all azimuthal angles becomes apparent. At this time the spacecraft is completely engulfed within the cusp.

Figures 6 and 7 show the distance calculated from the spacecraft to the cusp particle boundary and the respective orientation angles, using Eqs. (1) and (2). With this information, the approximate evolution of the boundary location with respect to the position of the spacecraft traveling at roughly $2.5 \mathrm{~km} / \mathrm{s}$ is illustrated in Fig. 8. The CEP boundary is observed for over $10 \mathrm{~min}$ as the trajectory of ISEE-1 takes the satellite outbound at a local time of $\sim 9 \mathrm{~h}(\Delta \mathrm{X} \sim \Delta \mathrm{Y})$. As the boundary is approached it appears to maintain a nearly constant location in $\mathrm{X}$ at about $5.08 R_{E}$ for less than two minutes as ISEE-1 approaches and then moves outward for the next seven minutes as ISEE-1 overtakes the boundary at 16:07:30 UT and crosses fully into the CEP region of the cusp. This boundary sounding technique reveals a dynamic boundary that could be related to changes in the solar wind conditions as well as the satellite motion.

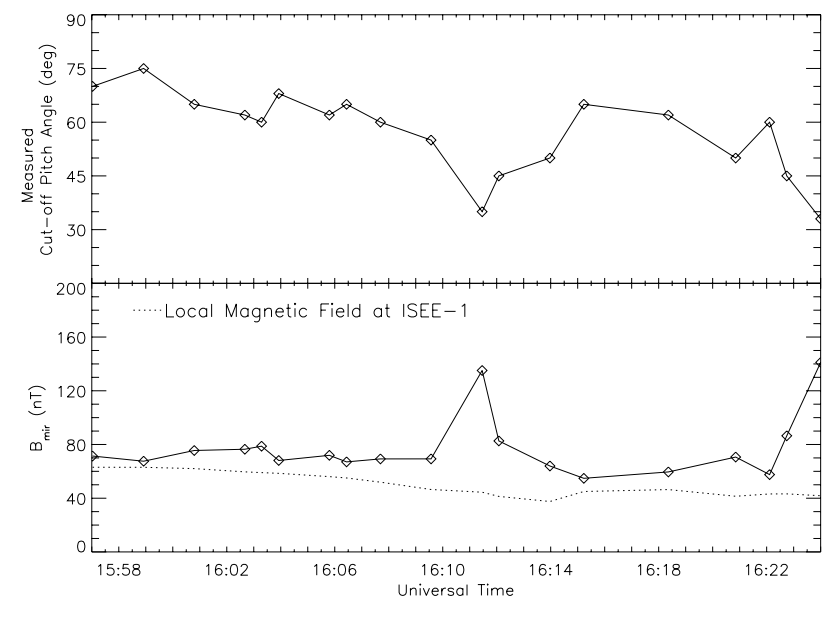

Fig. 9. The cut-off pitch angle is measured from 3-D PAD (top panel), where the s/c almost never observes particles with pitch angles less than 30 degrees. The magnitude of the corresponding mirror points for these particles are plotted in the bottom panel, where these values are approximately that of the LMF present outside the $\mathrm{CDC}(\sim 57 \mathrm{nT})$.

\section{Source of the CEP fluxes}

There appears to be a limiting pitch angle below which particles very rarely arrive in Fig. 3 . From this cut-off pitch angle, $\alpha_{\text {cutoff }}$, one can calculate the mirror point of the magnetic field, $B_{\text {mirror }}$, depending on the local magnetic field value,

$B_{\text {mirror }}=\frac{B_{\text {local }}}{\sin ^{2} \alpha_{\text {cutoff }}}$

In Fig. 9, $\alpha_{\text {cutoff }}$ is measured from the 3-D PAD from 15:5716:24 UT, and the magnitude of $B$ at the corresponding mirror points for these particles is calculated. The magnetic field values required for the particles to mirror are approximately that of the local magnetic field (LMF) present outside the $\mathrm{CDC}$ and therefore it is possible for many particles that originated in the cusp from below with pitch angles from $\alpha_{\text {cutoff }}$ to $\alpha$ near $90^{\circ}$ to mirror on a field line just outside and above the CDC and return back. This process can explain why a few particles are observed arriving from above the spacecraft. However, the most striking feature of the upper hemisphere remains the lack of particles.

As the spacecraft exits the cusp, a strong population is observed from below. The three-dimensional particle distributions are projections of the unit sphere, linearly translated from elevation and azimuth onto an orthogonal Cartesian coordinate system. Thus, enhancements at bottom of the panel will correspond to a small point at the bottom of a sphere. This type of projection exagerates the southern and northernmost points of a sphere. Through investigating the projection effect, the population is found to depend on the magnetic field. Particles hang on the $120^{\circ}$ PA contour after the 
spacecraft has exited the cusp and no longer observes a turbulent and depressed field. The source of particles from $120^{\circ}$ after ISEE-1 has left the cusp can be used to trace back to a rough altitude of origin.

If particles are being accelerated at some altitude within the cusp, one can estimate this location by assuming the particles are introduced with a pitch angle of $90^{\circ}$ at the mirror point and the spacecraft observes them predominantly peaking near $120^{\circ}$. This yields a mirror point at a local magnetic field value of $57 \mathrm{nT}$. In Fig. 1, the LMF has a value of about $57 \mathrm{nT}$ as the spacecraft first crosses into the depressed field region of the cusp.

\section{Discussion}

The observations from ISEE-1 indicate that the equatorial orbiting spacecraft passed through the cusp at a GSM Z of 6.0$6.8 R_{E}$, nominally observing the characteristic signatures in the proton and electron flux enhancements (known as CEPs), the local magnetic field depression with greatly enhanced turbulence, the presence of solar wind plasma, and a difference in the magnetic field clock angle comparison (Whitaker et al., 2006).

Work done by Whitaker et al. (2006) on this event has demonstrated that the CEP fluxes cannot originate from the geomagnetic tail through a substorm. Subsequently, the bow shock cannot be the main source of these particles either. If the bow shock was the source, a large population of particles would be observed from above. The deficit in particles with pitch angles less than about $60^{\circ}$ clearly shows that the bow shock is not providing the main source of the CEPs (see Figs. 3-5). The particles originate locally indicating that the source of CEPs could be from the dayside magnetospheric, or even within the cusp.

The observation of energetic particles after the spacecraft had exited the CDC and was above the cavity supports the theory of local energization. The Tsyganenko T04 model of the geomagnetic field shows open field lines for the entire orbit of ISEE-1 through the cusp. The last closed field line occurs between 15:50 UT and 16:00 UT, just before the spacecraft penetrates the CDC. The particles streaming from below are therefore lost on these open field lines and must be generated locally to have flux enhancements that persist for more than two hours. In future work, modeling of the evolution of the geomagnetic field and the particle trajectories along the trajectory of ISEE- 1 from a stable trapping region around 12:00 UT out through the cusp from 16:00-18:30 UT will be studied.

The length of time that ISEE-1 observes the CDC can be used to put a lower limit to the size of the cavity. ISEE-1 travels $4 R_{E}$ from 16:00-18:30 UT, suggesting that CDCs are very large, coherent structures. These observations by ISEE1 show a $>4 R_{E} \mathrm{CDC}$ that maintains CEPs of ring current energies during a moderate storm period ( $D_{s t}$ of $\left.-80 \mathrm{nT}\right)$ for greater than $2 \mathrm{~h}$.

Taylor et al. (2004) determined the orientation and velocity of the cusp boundary for a single pass by the Cluster satellites, detecting the boundary multiple times. Taylor et al. use two analysis techniques, the Fluxgate Magnetometer (FGM) Discontinuity Analyser and the Plasma Electron and Current Experiment (PEACE) Timing Analysis, which result in an inconsistent boundary orientation for a couple of the detections, where Cluster encounters the cusp from the poleward edge but exits equatorward. The boundary sounding technique used in this paper appears to produce a consistent time series set of boundary positions and orientations in a twodimensional geometry, owing to the snapshot nature of the technique. Taylor et al. have used two techniques that compare data recorded at different times and yield inconsistent results, implying that the variability of the cusp boundary in time can compromise the accuracy of the Cluster techniques.

\section{Conclusions}

ISEE-1 observes a CDC for over $2 \mathrm{~h}$ on 30 October 1978 during a moderate storm period. From the analysis of the CDC geometry and the CEP 3-D pitch angle distribution evolution, we find that:

- ISEE-1 is on open field lines in the dayside cusp during this entire time period, where fluxes measured by the satellite are observed to maintain a large anisotropy along the magnetic field direction with fluxes arriving primarily from below or earthward of the satellite and are not observed to return from above or sunward of the satellite.

- The energetic particles in this region maintain ring current energies for greater than $2 \mathrm{~h}$.

- ISEE-1 is moving toward an emitting cusp boundary, observing that the boundary to satellite distance decreases with time. The position of the boundary appears to be initially constant in $\mathrm{X}$ for less than two minutes but then moves outward such that the satellite appears to travel approximately parallel to it for about seven minutes before the satellite catches the boundary and crosses into the cusp and is completely engulfed by CEP fluxes. The orientation of the boundary normal during this interval varies through angular differences of $\sim 60$ degrees, but basically maintains an orientation approximately perpendicular to $\mathrm{X}$, indicating that the structure of the boundary described above is due to the outbound movement of the boundary as it is being sampled versus a fixed location in space that happens to be parallel to the trajectory of ISEE-1.

- After ISEE-1 no longer observes a depressed and turbulent magnetic field, the particles are still observed 
streaming from below along open field lines, where the $\mathrm{CDC}$ remains below the s/c.

- These observations show the main source of the energetic particles cannot be the bow shock.

These observations provide a challenge to the scientific community to investigate the possibility of a new region of interest at mid-altitudes in the cusp, where CDCs can produce ring current energy particles for long durations of time.

Acknowledgements. We thank G. K. Parks, G. L. Siscoe, and P. Song for useful discussions. Also, we thank E. S. Beiser and G. M. Gugliotti for their development of data analysis software for the 3-D pitch angle plots. This research was supported in part by NASA grants NAG5-2578, NAG5-7677, NAG5-7841, NAG5-9562, and NAG5-11397.

Topical Editor I. A. Daglis thanks two referees for their help in evaluating this paper.

\section{References}

Antonova, A. E. and Shabansky, V. P.: Particle and magnetic field in the outer dayside geomagnetosphere, Geomagn. Aeron., 15(2), 297-302, 1975.

Antonova, A. E., Gubar, Yu. I., and Kropotlin, A. P.: Energetic particle population in the high-latitude geomagnetosphere, Phys. Cham. Earth (C), 25(1-2), 47-50, 2000.

Asikainen, T. and Mursula, K.: Energetic particle fluxes in the exterior cusp and the high-latitude dayside magnetosphere: statistical results from the Cluster/RAPID instrument, Ann. Geophys., 23, 2217-2230, 2005,

http://www.ann-geophys.net/23/2217/2005/.

Asikainen, T. and Mursula, K.: Reconnection and energetic particles at the edge of the exterior cusp, Ann. Geophys., 24, 19491956, 2006, http://www.ann-geophys.net/24/1949/2006/.

Blake, J. B.: Comment on "Cusp: A new acceleration region of the magnetosphere" by Jiasheng Chen et al., Czech. J. Phys., 49(4a), 675-677, 1999.

Chang, S.-W., Scudder, J. D., Fuselier, S. A., Fennell, J. F., Trattner, K. J., Pickett, J. S., Spence, H. E., Menietti, J. D., Peterson, W. K., Lepping, R. P., and Friedel, R.: Cusp energetic ions: A bow shock source, Geophys. Res. Lett., 25, 3729-3732, 1998.

Chen, J., Fritz, T. A., Sheldon, R. B., Spence, H. E., Spjeldvik, W. N., Fennell, J. F., and Livi, S.: A new, temporarily confined population in the polar cap during the August 27, 1996 geomagnetic field distortion period, Geophys. Res. Lett., 24, 1447-1450, 1997.

Chen, J. and Fritz, T. A.: Correlation of cusp MeV helium with turbulent ULF power spectra and its implications, Geophys. Res. Lett., 25, 4113-4116, 1998.
Chen, J., Fritz, T. A., Sheldon, R. B., Spence, H. E., Spjeldvik, W. N., Fennell, J. F., Livi, S., Russell, C. T., Pickett, J. S., and Gurnett, D. A.: Cusp energetic particle events: Implications for a major acceleration region of the magnetosphere, J. Geophys. Res., 103(A1), 69-78, 1998.

Delcourt, D. C. and Sauvaud, J.-A.: Populating of the cusp and boundary layers by energetic (hundreds of $\mathrm{keV}$ ) equatorial particles, J. Geophys. Res., 104, 22 635-22 648, 1998.

Farrell, W. M. and Van Allen, J. A.: Observations of the Earth's polar cleft at large radial distances with the Hawkeye 1 magnetometer, J. Geophys. Res., 95, 20 945-20 958, 1990.

Fritz, T. A. and Fahnenstiel, S. C.: High Temporal Resolution Energetic Particle Soundings at the Magnetopause on November 8 , 1977, Using ISEE 2, J. Geophys. Res., 87, 2125-2131, 1982.

Fritz, T. A., Chen, J., and Siscoe, G. L.: Energetic ions, large diamagnetic cavities, and Chapman-Ferraro cusp, J. Geophys. Res., 108(A1), 1028, doi:10.1029/2002JA009476, 2003.

Fung, S. F., Eastman, T. E., Boardsen, S. A., and Chen, S.-H.: Highaltitude cusp positions sampled by the Hawkeye satellite, Phys. Chem. Earth, 22, 653-662, 1997.

Konradi, A. and Kaufmann, R. L.: Evidence for rapid motion of the outer boundary of the magnetosphere, J. Geophys. Res., 84, 1627-1637, 1965

Kremser, G., Woch, J., Mursula, K., Tanskanen, P., Wilken, B., and Lundin, R.: Origin of energetic ions in the polar cusp inferred from ion composition measurements by the Viking satellite, Ann. Geophys., 13, 595-607, 1995, http://www.ann-geophys.net/13/595/1995/.

Shabansky, V. P.: Some processes in the magnetosphere. Space Sci. Rev., 12, 299-418, 1971.

Taylor, M. G. G. T., Dunlop, M. W., Lavraud, B., Vontrat-Reberac, A., Owen, C. J., Décréau, Trávníček, P., Elphic, R. C., Friedel, R. H. W., Dewhurst, J. P., Wang, Y., Fazakerley, A., Balogh, A., Rème, H., and Daly, P. W.: Cluster observations of a complex high-altitude cusp passage during highly variable IMF, 22, $3707-$ 3719, 2004.

Trattner, K. J., Fuselier, S. A., Peterson, W. K., Chang, S.-W., Friedel, R., and Aellig, M. R.: Origins of energetic ions in the cusp, J. Geophys. Res., 106, 5967-5976, 2001.

Whitaker, K. E., Chen, J., and Fritz, T. A.: CEP populations observed by ISEE 1, Geophys. Res. Lett., 33, L23105, doi:10.1029/2006GL027731, 2006.

Williams, D. J., Keppler, E., Fritz, T. A., Wilken, B., and Wibberenz, G.: The ISEE 1 and 2 Medium Energy Particles Experiment, IEEE Trans. Geosci. Electron., 16, 270-280, 1978.

Williams, D. J.: Magnetopause characteristics inferred from threedimensional energetic particle distributions, J. Geophys. Res. 84, 101-104, 1979. 